\title{
Red neuronal convolucional aplicado a la estimulación de motricidad fina con un videojuego en un paciente con hemiparesia
}

\section{Convolutional neural network applied to fine motor stimulation with a video game in a patient with hemiparesis}

Tipantocta Fabricio Instituto Superior Tecnológico Sucre, Ecuador ftipantocta@tecnologicosucre.edu.ec

Rosero Ricardo Instituto Superior Tecnológico Sucre, Ecuador rrosero@tecnologicosucre.edu.ec

Velasco Eduardo Instituto Superior Tecnológico Sucre, Ecuador evelasco@tecnologicosucre.edu.ec

Tipan Flor Instituto Superior Tecnológico Sucre, Ecuador ftipan@tecnologicosucre.edu.ec

\section{Revista Cumbres Vol.6 №1}

Versión electrónica ISSN 1390-3365 http://investigacion.utmachala.edu.ec/revistas/index.php/Cumbres 


\title{
RESUMEN
}

El objetivo del proyecto es desarrollar un videojuego, implementando algoritmos de una red neuronal convolucional con aprendizaje profundo para el reconocimiento del movimiento de la mano en un paciente con hemiparesia izquierda y así realizar acciones de control con la finalidad de rehabilitar a la persona. Se entrenó la red neuronal convolucional con 600 imágenes de manos abiertas y 600 imágenes de manos cerradas del niño con hemiparesia izquierda, clasificando así las posibles soluciones para realizar acciones de control y realizar el enlace con un videojuego realizado en la plataforma Unity. Como resultado se tiene un porcentaje de probabilidad del 99\% cuando la mano izquierda se encuentra abierta o cerrada, enviando comandos para que el videojuego funcione; de esta manera el paciente con hemiparesia, a la necesidad de abrir y cerrar su mano izquierda ejecuta acciones de control y provoca por sus propios medios su motricidad y rehabilitación. Al finalizar las pruebas el niño se sentía cansado por el hecho de que el sistema le incentivaba a mover su brazo, ya que generalmente esa extremidad no la movía mucho, lo que se puede concluir que el sistema funciona correctamente, cumpliendo su objetivo.

Palabras clave: videojuego, biomecánica, hemiparesia, red neuronal convolucional.

\begin{abstract}
The objective of this project is to develop a video game for the recognition of the movement of the hand in a patient with left hemiparesis and thus perform control actions in order to rehabilitate the person. Algorithms of a convolutional neural network with deep learning were implemented. The network was trained with 600 images of open hands and 600 images of closed hands of a child with left hemiparesis. This allowed the classification of possible solutions to perform control actions and link to a video game on the Unity platform. The results show that there is a 99\% probability for sending commands for the video game to work when the left hand is open or closed. In this way, when the patient with hemiparesis needs to open and close his left hand, he executes control actions and provokes his mobility and rehabilitation. At the end of the tests, the child felt tired by the fact that the system encouraged him to move his arm. Generally, he did not move that limb that much. It can be concluded that the system works correctly, fulfilling its objective.
\end{abstract}

Keywords: video game, biomechanics, hemiparesis, convolutional neural network.

\section{Cumbres}




\section{INTRODUCCIÓN}

La parálisis cerebral infantil (PCI) a nivel global está entre un 2 y un 3 por cada 1000 nacidos vivos y actualmente sigue en aumento.(Monteagudo, 2016) Una forma de presentarse la PCI es la hemiparesia, la cual afecta una hemiparte del cuerpo siendo esta congénita o adquirida.(Espin, 2014) Para el análisis de este proyecto, se toma en cuenta a un niño de 7 años con hemiparesia izquierda, quien después de haber recibido un golpe en la cabeza al nacer le originó un coagulo de sangre lo cual originó dicha patología. Teniendo así que rehabilitar su lado afectado con terapias físicas para ayudar al movimiento de afectado de sus miembros, en especial su brazo y mano izquierda.

Los pacientes con hemiparesia presentan trastornos tróficos, y denota un retraso en el crecimiento de extremidades y el proceso para rehabilitar al paciente depende del nivel de afectación en el sistema nervioso. Siempre que sea posible, los pacientes requieren una amplia variedad de servicios profesionales que demandan gran coordinación y esfuerzo con el fin de obtener los mejores resultados. (Gutierrez, Reyes, Tovar, Alzate, \& Bohórquez, 2007) (Jover Torres, 2016) Actualmente el paciente escogido, ha pasado por una serie de terapias para mejorar su condición física, pero en la actualidad únicamente asiste al programa de rehabilitación ocupacional donde se hace énfasis en la realización de tareas y actividades de la vida diaria.

Existen varios tratamientos en la rehabilitación ocupacional, una de ellas es el sistema "Mirror-Neuron".(Tipantocta, 2019) Esta técnica hace referencia a la estimulación del sistema nervioso en base al trabajo de aprendizaje por reflejo, el cual por medio de observación de actividades pretende que la persona afectada realice los mismos movimientos en brazos y piernas. (Yuan \& Hoff, 2008)(Rizzolatti \& Craighero, 2016) Con este tratamiento de rehabilitación, y observando la preferencia del paciente por la tecnología digital como teléfonos celulares, tablets y televisión, se ideo la forma de que el paciente con hemiparesia, se interese y estimule el sistema motor de la mano izquierdo mediante la realización de un videojuego. (Universidad de Guadalajara, 2016)

El objetivo del proyecto es desarrollar un videojuego, implementando algoritmos de aprendizaje profundo para el reconocimiento del movimiento de la mano en un paciente con hemiparesia izquierda y así realizar acciones de control con la finalidad de rehabilitar a la persona.

\section{MATERIALES Y METODOS}

\section{Biomecánica de la mano}

La hemiparesia afecta a extremidades de un lado del cuerpo. Existe disminución del tono muscular en el hemicuerpo afectado, que para el caso de análisis de este estudio es el brazo izquierdo del paciente. Otra característica 
en casos severos es la espasticidad que afecta a ciertas cadenas musculares como los flexores del brazo, el cual presenta un patrón típico de aducción de hombro, flexión de codo, pronación de antebrazo, flexión de muñeca y dígitos con aducción del pulgar. (Murie-Fernández et al., 2012) Por este motivo, con el paso del tiempo el paciente podría tener dolor muscular por falta de movilidad en la mano, muñeca y brazo.

Según (Repetto, 2007) (Diaz-hernandez, 2017), el objetivo principal de la biomecánica es evaluar la relación entre el movimiento ejecutado y el gasto de energía implicado en su realización. Para el proyecto se analiza el movimiento de la muñeca, el cual se efectúa con la mano y se realizan los movimientos de flexo-extensión.(Hirsch \& Avaria, 2015)

Figura 1. a) Extensión activa de 85, y b) Flexión extensión de muñeca

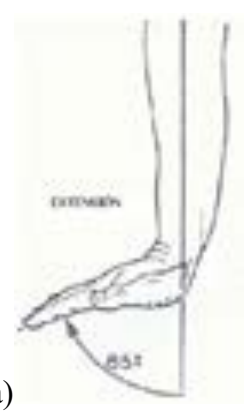

b)

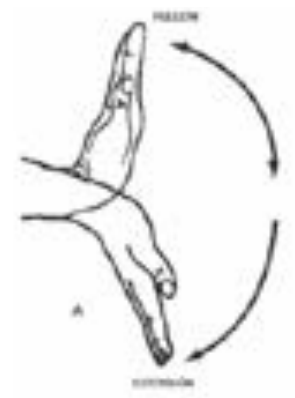

Este movimiento presentado en la figura 1, se conoce como flexión-extensión de muñeca, el cual se encuentra en el plano sagital, en el eje transversal y la flexión y extensión llega hasta los 85. (Diaz-hernandez, 2017) Conociendo la afectación que provoca la hemiparesia en el niño, el cual no utiliza mucho su mano izquierda, denota crecimiento tardío en su brazo izquierdo con respecto al derecho por mantener mucho su mano izquierda en posición cerrada, se puede apreciar en la figura 2.

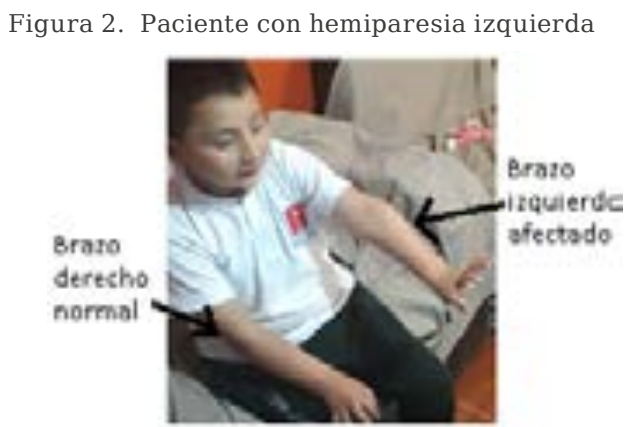

Sabiendo estas características, se idea la forma de que el paciente abra su mano de una forma natural y que por sus propios medios realice su rehabilitación, para lo cual se entrena un sistema de red neuronal con aprendizaje profundo para aprender cuando la mano del paciente este abierto (extensión) o cerrado por reconocimiento visual, esto se explica en el siguiente 


\section{Red neuronal convolucional (CNN)}

El Deep Learning o aprendizaje profundo está transformando y a la vez revolucionando el campo de la visión artificial.(Chanampe et al., 2019) El uso de imágenes como dato de entrada, da la apertura a la teoría de la red neuronal convolucional el cual maneja una gran cantidad de información para las neuronas de entrada. Según (DURÁN, 2017), una red neuronal convolucional es un tipo de red multicapa que consta de diversas capas convolucionales y de pooling (submuestreo) alternadas, y al final tiene una serie de capas full-connected como una red perceptron multicapa; en la figura 3 se representa el modelo de las capas en la red neuronal convolucional CNN.

Figura 3. Esquema básico de una red neuronal convolucional CNN

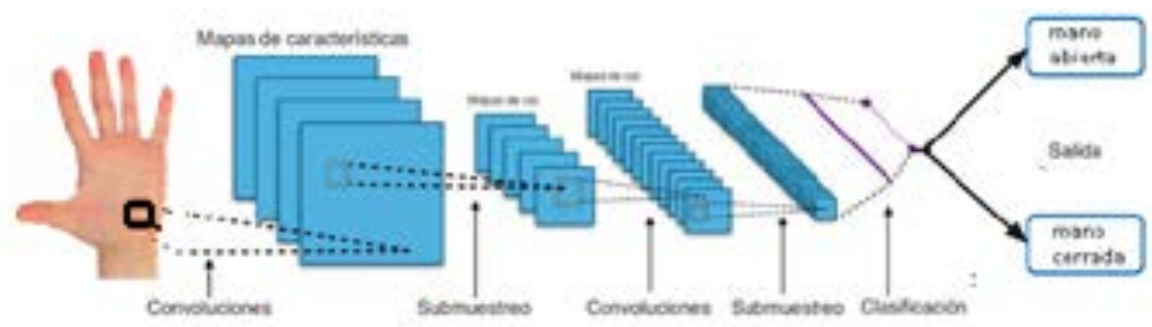

Para el proyecto se planteó entrenar la CNN con métodos convencionales como es la librería Tensorflow de Google y el entorno de anaconda 3.5, en una PC con Windows 10 y una GPU Nvidia Gforce para acelerar el proceso de entrenamiento de la red.

Para entrenar la red, se estimó colocar un set de 600 imágenes de manos abiertas y 600 imágenes de manos cerradas del paciente con hemiparesia de $640 * 480$ pixeles con 3 canales RGB de entrada para obtener el resultado de condición de una mano abierta y una mano cerrada. Para controlar la librería Tensorflow se usó el código desarrollado por google en Python con la plataforma Anaconda 3.5.

\section{Videojuegos con Unity}

La industria de los videojuegos ha crecido impresionantemente en los últimos años y países europeos como en España, disfrutan de este tipo de entretenimiento siendo este el sexto a nivel mundial. (Universidad de Guadalajara, 2016) (Cuesta, Cuesta, \& Rodríguez-Osorio, 2014) Para el proyecto se ideo trabajar con un videojuego por el interés que se tiene en la actualidad y escoger una plataforma que permita crear videojuegos atractivos para niños puede ser una tarea complicada, por tal motivo se escogió la plataforma Unity por su utilidad a la hora de realizar aplicaciones.(Aymerich Franch, 2013) (Fuentes, 2011) 
Para el diseño del videojuego adaptado al paciente, y teniendo presente su edad de 7 años, se crea un ambiente de fantasía en 2 dimensiones, en el cual se incorpora un jugador el cual tiene la capacidad de correr y saltar por el escenario creado y teniendo que evadir enemigos que aparecerán cada 10 segundos, esto se muestra en la figura 4.

Figura 4. Juego diseñado en Unity.

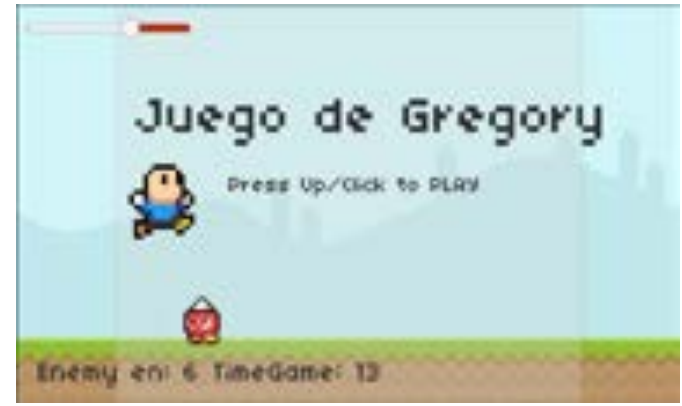

\section{RESULTADOS}

\section{Entrenamiento de la red neuronal convolucional (CNN)}

Para entrenar la red se tomó muestras de 600 imágenes con la mano abierta (extensión) y 600 imágenes con la mano cerrada. Estas imágenes se usaron como valores de entrada al ejecutar el entrenamiento, el clasificador de tensorflow ejecuta paso a paso el algoritmo de aprendizaje de las imágenes, hasta encontrar el error más cercano a 0, en la figura 5 muestra el algoritmo de aprendizaje con 600 pasos y con 12000 pasos; este denota como el algoritmo tiene tendencia a ser 0 cuando se ejecuta mayor número de pasos de aprendizaje.

Figura 5. Evolución del algoritmo de aprendizaje con 600 y 12000 pasos
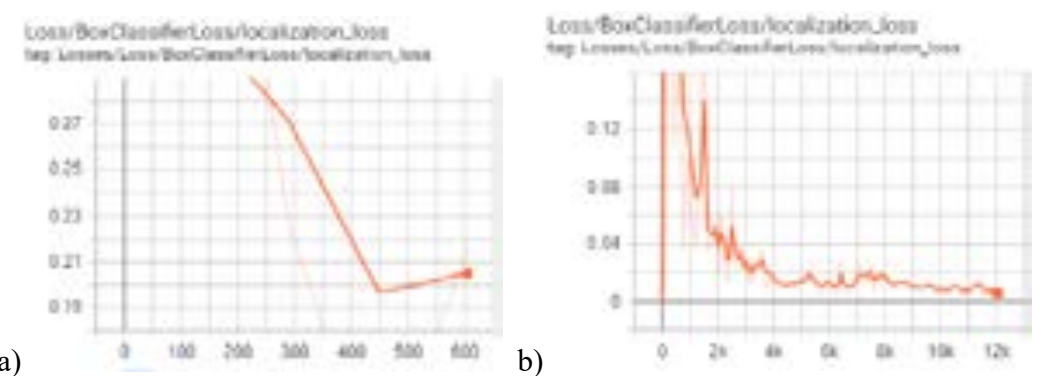

Con el error al $0.005 \%$, transcurrido tres horas y 12000 pasos se corta el funcionamiento del algoritmo de aprendizaje y la red se encuentra entrenada; cabe especificar que existe un máximo número de pasos que es 200.000, pero el error estaría bordeando los mismos valores. Como resultado del entrenamiento de la red se presenta en la figura 6. 
Figura 6. Resultado del entrenamiento de la red neuronal convolucional.
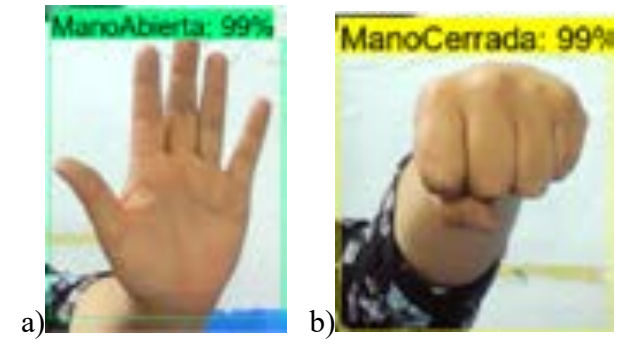

Como resultado se tiene un porcentaje de probabilidad del 99\% cuando una mano se encuentra abierta (en extensión) o cerrada. Con estos valores se puede realizar acciones de control, el cual se lo utilizará en la aplicación del videojuego desarrollado en Unity.

\section{Aplicación de la CNN en el videojuego}

Para este proyectos se utilizó dos tipos de programas como son: Python para la CNN y Unity para el videojuego; y para poder conectar los datos entre programas se usó un comunicador serial por puertos virtuales COM. Por tal motivo se tiene que ejecutar como primera instancia el videojuego y luego se ejecuta la CNN, con esto el paciente estará listo para jugar, se aprecia en la figura 7.

Figura 7. Paciente jugando con el sistema incorporado

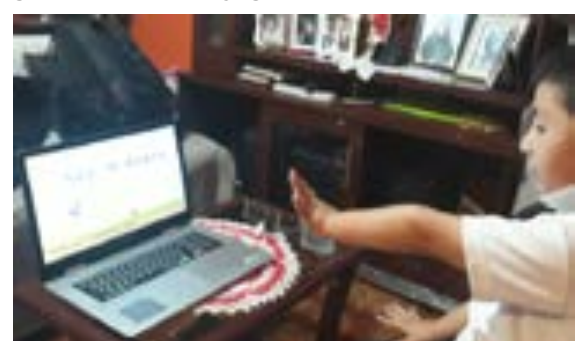

Se examina la extensión de la mano y el cierre de la misma con el software de análisis de movimiento Tracker. El videojuego reacciona a la apertura de la mano, haciendo que el jugador salte en el ambiente creado, tomando en cuenta que a los 10 segundos empezará a salir un enemigo que deberá evitar con un salto.

Figura 8. Movimiento del brazo, cambiando inclinación del sensor.
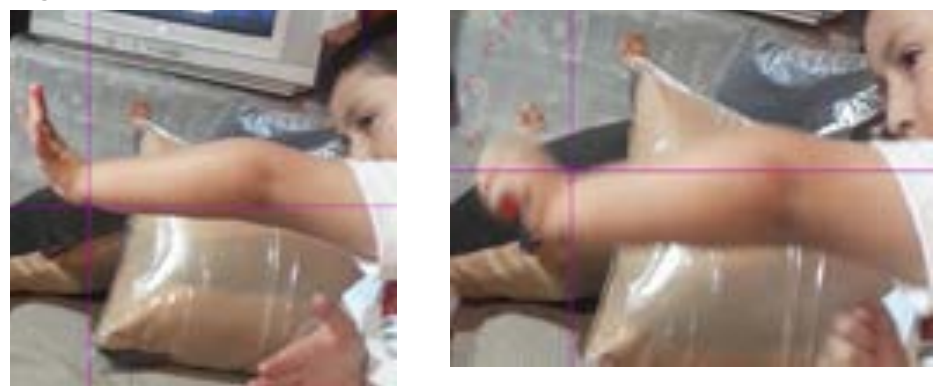
Se toma en cuenta este primer análisis en tracker observando los datos recogidos en la tabla 1, el cual toma valores de velocidad angular y posición.

Tabla 1. Velocidad angular y posición de la mano

\begin{tabular}{|l|l|l|}
\hline T(SEG) & $\mathbf{\Omega}\left({ }^{\circ} / \mathbf{S E G}\right)$ & $\mathbf{X}(\mathbf{M})$ \\
\hline 5,7665 & $5,54 \mathrm{E}+01$ & $-2,84 \mathrm{E}-01$ \\
\hline 5,7670 & $1,31 \mathrm{E}+02$ & $-2,52 \mathrm{E}-01$ \\
\hline 5,7675 & $2,40 \mathrm{E}+02$ & $-2,26 \mathrm{E}-01$ \\
\hline 5,7680 & $3,67 \mathrm{E}+02$ & $-1,62 \mathrm{E}-01$ \\
\hline 5,7685 & $4,30 \mathrm{E}+02$ & $-5,31 \mathrm{E}-02$ \\
\hline 5,7690 & $3,47 \mathrm{E}+02$ & $2,58 \mathrm{E}-02$ \\
\hline 5,7695 & $2,12 \mathrm{E}+02$ & $6,07 \mathrm{E}-02$ \\
\hline
\end{tabular}

Observando como el paciente, a la necesidad de que el jugador salte, realiza movimientos de apertura y cierre y se puede apreciar la velocidad máxima de $400 \mathrm{rev} / \mathrm{seg}$ con el cual ejecuta la acción.

Figura 9. Gráfica de velocidad angular y posición de la mano izquierda.

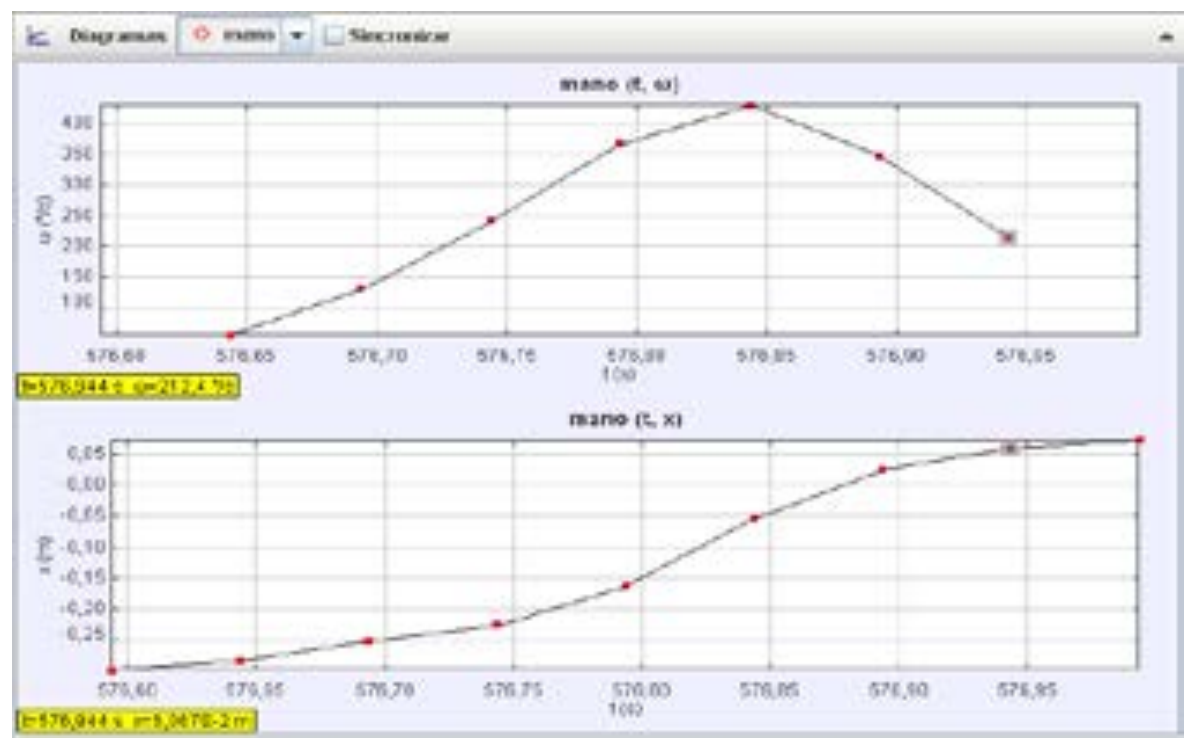

El niño estimula por sí mismo la motricidad, ya que debe abrir y cerrar su mano por sus propios medios. Tomando en cuenta las recomendaciones del neurólogo de cabecera, sugirió realizar pruebas cortas y se tomó como parámetro estimar un tiempo de prueba de 5 minutos, y como máximo 5 pruebas por día, dado este parámetro y sabiendo que el enemigo sale cada 10 segundos se toma un estimado de 30 puntos si el paciente brincara todos, la tabla 2 muestra las pruebas realizadas en un día al azar 
Tabla 2. Pruebas de funcionamiento

\begin{tabular}{|l|l|l|l|l|}
\hline VARIABLE & OBS & PROMEDIO & STD. DEV. & CONTEOPUNTOS \\
\hline P1 & 30 & .4 & .4982729 & 12 \\
\hline P2 & 30 & .3666667 & .4901325 & 11 \\
\hline P3 & 30 & .5333333 & .5074163 & 16 \\
\hline P4 & 30 & .6333333 & .4901325 & 19 \\
\hline P5 & 30 & .6666667 & .4794633 & 20 \\
\hline
\end{tabular}

Como resultado en esta serie de experimentos, se muestra que entre la prueba 4 y la prueba 5 adquiere mayor experiencia, obteniendo un mayor puntaje. Se observa el trabajo realizado por la mano izquierda que es el objetivo del proyecto, estimulando así su motricidad.

\section{CONCLUSIONES}

Se desarrolló el video juego implementando un algoritmo de red neuronal convolucional CNN para la rehabilitación de un niño con hemiparesia izquierda. El niño se motiva mucho al jugar con el videojuego, realizando en momentos movimientos bruscos para abrir la mano, lo que demuestra que la aplicación funciona correctamente, pero cabe destacar las recomendaciones del neurólogo ya que esto podría repercutir en pacientes que tengan tendencia a tener epilepsia.

El sistema actualmente necesita una gran capacidad de procesamiento por lo cual la PC usada debe tener una tarjeta de video para que pueda correr el programa, pero se puede bajar mucho más si se baja la calidad de las imágenes al momento de entrenar la red, si no se necesita características grandes que aprenda la red.

Al finalizar las pruebas el niño se sentía cansado por el hecho de que el sistema le incentivaba a mover su brazo, ya que generalmente esa extremidad no la movía mucho, lo que se puede concluir que el sistema funciona correctamente, cumpliendo su objetivo.

\section{REFERENCIAS BIBLIOGRÁFICAS}

Aymerich Franch, L. (2013). La realidad virtual como herramienta de estudio de fenómenos psicológicos y sociales. Orbis. Revista Científica Ciencias Humanas.

Chanampe, H., Aciar, S., Vega, M. De, Luis, J., Sotomayor, M., Carrascosa, G., \& Lorefice, A. (2019). Modelo de Redes Neuronales Convolucionales Profundas para la Clasificación de Lesiones en Ecografías Mamarias. XXI Workshop de Investigadores En Ciencias de La Computación, (Cidi). 
Cuesta, D. B., Cuesta, B. G., \& Rodríguez-Osorio, J. J. (2014). Videojuego 3D en Unity destinado al aprendizaje del Alfabeto dactilológico, 1-162. Retrieved from http://eprints.ucm.es/26491/1/ProjectNewDetroit_Tecnología e Implementación_Memoria_UCM.pdf

Diaz-hernandez, O. (2017). Un análisis cinemático del brazo humano para biomecánica, (September 2014).

DURÁN, J. (2017). Redes Neuronales Convolucionales en R: Reconocimiento de caracteres escritos a mano. Universidad de Sevilla. Universidad de sevilla, Sevilla.

Espin, S. (2014). Hemiparesia izquierda a consecuencia de accidente de tránsito en adolescentes de 16 años. Repo.Uta.Edu.Ec, 115. https://doi. org/10.1002/14651858.CD010789.

Fuentes, J. (2011). “ Diseño E Implementación De Un Videojuego Multiplayer Online , Utilizando El Motor Unity 3d”, 20-30.

Gutierrez, D. J., Reyes, D. C. A., Tovar, D. M., Alzate, D. N., \& Bohórquez, D. F. (2007). Rehabilitacion En Trauma Craneoencefálico. Convenio ISS- ASCOFAME. https://doi.org/10.1177/0149206315573998

Hirsch, M., \& Avaria, P. (2015). Músculos accesorios de la muñeca : Revisión pictográfica en US y RM. Revista Chilena de Radiología, 21(1), 138-143.

Jover Torres, M. (2016). Rehabilitación del miembro superior mediante Terapia de movimiento inducido por restricción del lado sano en paciente neurológico., 30. Retrieved from http://dspace.umh.es/bitstream/11000/2988/1/ JOVER TORRES, MIRIAM.pdf

Monteagudo, P. (2016). Eficacia De La Terapia Intensiva Bimanual En Niños Con Hemiparesia. Universidad de Cantabria. Retrieved from https://repositorio.unican.es/xmlui/bitstream/handle/10902/9992/Monteagudo Jato $\% 2 \mathrm{C}$ Patricia.pdf?sequence $=1 \&$ is Allowed $=\mathrm{y}$

Murie-Fernández, M., Carmona Iragui, M., Gnanakumar, V., Meyer, M., Foley, N., \& Teasell, R. (2012). Hombro doloroso hemipléjico en pacientes con ictus: Causas y manejo. Neurologia, 27(4), 234-244. https://doi.org/10.1016/j. nrl.2011.02.010

Repetto, A. (2007). Bases biomecánicas para el análisis del movimiento humano. Revista ITSS SUcre, 201-210.

Rizzolatti, G., \& Craighero, L. (2016). The Mirror-Neuron System. Family Medicine, 48(9), 731. https://doi.org/10.1146/annurev.neuro.27.070203.144230

Tipantocta, F. (2019). Estimulación de motricidad fina con un videojuego en un paciente con hemiparesia izquierda. AVANCES EN BIOINGENIERÍA Y SISTEMAS INTELIGENTES DE REHABILITACIÓN EN LATINOAMÉRICA 2019, II(ISBN: 978-9942-784-66.), 97-100.

Universidad de Guadalajara, C. L. (2016). El videojuego como herramienta educativa. Apertura, 8(1), 136-151. Retrieved from http://www.udgvirtual. udg.mx/apertura/index.php/apertura/article/view/825/539

Yuan, T. F., \& Hoff, R. (2008). Mirror neuron system based therapy for emotional disorders. Medical Hypotheses, 71(5), 722-726. https://doi.org/10.1016/j. mehy.2008.07.004

\section{Cumbres}

